This item was submitted to Loughborough's Research Repository by the author.

Items in Figshare are protected by copyright, with all rights reserved, unless otherwise indicated.

\title{
Spatialization in working memory: can individuals reverse the cultural direction of their thoughts?
}

PLEASE CITE THE PUBLISHED VERSION

https://doi.org/10.1111/nyas.14499

PUBLISHER

Wiley

VERSION

AM (Accepted Manuscript)

\section{PUBLISHER STATEMENT}

This is the peer reviewed version of the following article: Guida, A., Mosinski, F., Cipora, K., Mathy, F. and Noël, Y. (2020), Spatialization in working memory: can individuals reverse the cultural direction of their thoughts?. Annals of the New York Academy of Sciences, 1477 (1), pp.113-125, which has been published in final form at https://doi.org/10.1111/nyas.14499. This article may be used for non-commercial purposes in accordance with Wiley Terms and Conditions for Use of Self-Archived Versions.

\section{LICENCE}

CC BY-NC-ND 4.0

\section{REPOSITORY RECORD}

Guida, Alessandro, Francis Mosinski, Krzysztof Cipora, Fabien Mathy, and Yvonnick Noel. 2020.

"Spatialization in Working Memory: Can Individuals Reverse the Cultural Direction of Their Thoughts?". Loughborough University. https://hdl.handle.net/2134/12906224.v1. 


\title{
Spatialization in working memory:
}

\section{Can individuals reverse the cultural direction of their thoughts?}

Alessandro Guida ${ }^{1}$, Francis Mosinski ${ }^{2}$, Krzysztof Cipora $^{3}$, Fabien Mathy $^{4}$, \& Yvonnick Noël $^{1}$

\author{
${ }^{1}$ LP3C, Department of Psychology, Université Rennes, France \\ ${ }^{2}$ Department of Life Sciences and the Environment, Université Rennes, France \\ ${ }^{3}$ Centre for Mathematical Cognition, Loughborough University, Loughborough, UK \\ ${ }^{4}$ Bases Corpus Langage UMR 7320 CNRS, Université Côte d'Azur, Nice, France
}

Running head: Reversing the SPoARC effect

Word Count: 6301

Address correspondence to:

Alessandro Guida

Laboratoire de Psychologie, Comportement, Cognition et Communication

Université Rennes 2 - Haute Bretagne

Place du Recteur Henri Le Moal CS 24307

35043 Rennes Cedex

Tél : 0033 (0)2.23.22.58.78

Emails: alessandro.guida@univ-rennes2.fr

alessandro.guida.psychology@gmail.com 


\begin{abstract}
A recent study based on the SPoARC effect (Spatial Position Association Response Codes) showed that culture heavily shapes our cognition and more specifically the way we organize our thoughts ; when Western adults are asked to keep in mind a sequence of colors, they mentally organize them from left to right, whereas right-to-left reading/writing adults spatialize them in the opposite direction. Here we investigate if the spontaneous direction of spatialization in Westerners can be reversed. Lists of 5 consonants were presented auditorily at a rate of $3 \mathrm{~s}$ per item, participants were asked to mentally organize the memoranda from right to left. Each list was followed by a probe. Participants had to indicate whether the probe was part of the sequence by pressing a "yes" key or a "no" key with the left or right index finger. Left/right-hand key assignment was switched after half of the trials were completed. Results showed a reverse SPoARC effect that was comparable in magnitude to the spontaneous left-to-right SPoARC effect found in a previous study. Overall, our results suggest that individuals can reverse the cultural direction of their thoughts.
\end{abstract}




\section{Introduction}

Spatialization can be defined as the addition by the cognitive system of a spatial dimension that is not part of the actual input. ${ }^{1}$ One of the most known spatialization is the SNARC effect (Spatial Numerical Association Response Codes), ${ }^{2}$ which is usually studied with parity and magnitude judgments performed on a number (usually single digits, e.g., the interval between 1 and 9). This effect shows that individuals tend to respond faster to relatively small numbers (e.g., $1,2)$ with the effector that operates in the left side of space (e.g. the index finger of the left hand) and faster responses to relatively large numbers (e.g., 8, 9) with the effector that operates in the right side of space (e.g., the index finger of the right hand). ${ }^{3}$ Dehaene et al. ${ }^{2}$ proposed that this effect is due to the representation numbers have in semantic memory that takes the form of a mental numerical line (for reviews, see Refs. 4-7), its direction and orientation varying according to cultural reading habits: it runs from left to right in Westerners and in the opposite direction in right-to-left reading cultures., ${ }^{2,8,9}$

More recently a new type of spatialization has been brought to light by van Dijck and Fias: ${ }^{10}$ the SPoARC (Spatial Positional Association Response Codes) effect—also known as the ordinal position effect ${ }^{11}$ (not to be mistaken with the ordinal position effect proposed by Becker ${ }^{12}$ ). Various paradigms can be used to observe the SPoARC effect (for a complete description see Ref. 6). Here, we present Guida et al. ${ }^{13}$ 's paradigm as we used a similar one in the present study — we believe that the outcome would be similar using other SPoARC paradigms such as the one used by van Dijck and Fias. ${ }^{10}$ Guida et al. ${ }^{13}$ presented auditorily sequences of 5 consonants in an item probe recognition paradigm. ${ }^{14}$ After each sequence, participants heard a sixth consonant and had to indicate if it belonged to the sequence by pressing a "yes" key with one hand or a "no" key with the other hand. Guida et al. ${ }^{13}$ adapted Sternberg's paradigm in order for the left-/right-hand key assignment to be varied: the answer "yes" (and the 
answer "no") was assigned for the first half of the trials to one hand and for the other half to the other hand. Results showed that the difference between right- and left-hand reaction times decreased (showing an increasing right-hand advantage) when progressing toward the last positions being probed. This link between space and position in working memory (WM) has been observed several times ${ }^{1,10,11,15-17}$ and is interpreted as if Westerners organize the memoranda through a mental line from left to right. Abrahamse et al. ${ }^{18}$ have proposed a theoretical framework to account for this effect: the mental whiteboard hypothesis. If the cognitive system is confronted with a sequence of verbal information that can be processed semantically, the cognitive system internally generates a spatial template onto which memoranda can be bound.

Beyond the SPoARC effect, the mental whiteboard ${ }^{19}$ or more generally space ${ }^{20,21}$ has been proposed as a means to code order. For instance, Bonato et al. ${ }^{3,22}$ suggested a common mechanisms of spatial representation for responses to both long and short-term ordered representations. Up to now, no consensus has emerged concerning the mechanism that would code order in memory (for reviews see Refs. 23,24). Several very different mechanisms have been proposed (e.g., 25-28) which will be developed in the discussion. Hence, shading light on the SPoARC effect should enable understanding the factors linking the representation of order with spatial responses.

The difference between the SNARC effect and the SPoARC effect is that in the former, the mental line (for example from 1 to 9 ) is thought to exist in long-term memory. It can be labelled long-term memory spatialization, although this category is heterogenous and more fine grain classifications can be found (e.g., Refs. 29, 30). Concerning the SPoARC effect, the mental line must be built on the fly in WM as the sequences are random (for example the 
sequence "L”, “F”, “D”, or “2”, “7”, “1”), hence a WM spatialization. Van Dijck and Fias ${ }^{10}$ first thought that the SPoARC effect could explain the SNARC effect, but more recent results argue in favor of separate effects. ${ }^{31,32}$

After more than thirty years of research, a lot is known about the SNARC effect whereas the SPoARC has not revealed all of its secrets. One interesting feature of the SNARC effect is its flexibility. When Western participants are asked to think about numbers along a clock $^{33}$ or to think about a reversed numerical mental line that runs from 1 to 9 from right to left, ${ }^{34}$ the SNARC effect in Westerners is reversed (for a review see Ref. 35). Currently it is not known if the SPoARC effect can be reversed in a similar fashion. What we do know in terms of direction, is that the SPoARC effect follows the reading/writing direction in literates whereas it has no specific direction in illiterates, ${ }^{36}$ which made Guida et al. ${ }^{36}$ suggest that culture profoundly shapes the way we organize our thoughts.

By means of this study, we wanted to fill a gap of knowledge concerning our capacity to reverse the cultural direction of our thoughts. For this purpose, we used the same auditory paradigm than Guida et al. ${ }^{13}$ with the exception that we asked our participants to imagine the consonants from right to left. Based on the mental whiteboard hypothesis, ${ }^{18}$ we made the a-priori hypothesis that the participants would be able to reverse the SPoARC effect, as one of the assumptions of this framework is that space can be flexibly recruited for the coding of serial order, as long as it involves an orderly, well-arranged spatial dimension (i.e., in this experiment would be a right-to-left mental line). Finally, we compared the magnitude of the SPoARC effect in the present study and in Guida et al., ${ }^{13}$ to test if the flexibility would be so extreme that there would be no difference. We did not have a strong a-priori hypothesis concerning this last comparison as we were venturing in uncharted territory. We only knew from a previous 
SPoARC study ${ }^{37}$, that when items are displayed on a screen from left to right or from right to left, the SPoARC effect reverses but the magnitude of the effect remains the same.

\section{Method}

\subsection{Participants}

The participants were 30 students (18 females and 12 males) from Rennes University. All were native French speakers (one left-handed, mean age 22.7 years, $S D=1.79$ ).

\subsection{Material}

The material was identical to that of Guida et al. ${ }^{13}$. Participants were given two lists of 36 sequences of five different consonants. The sequences were created by pseudo-randomly sampling consonants without replacement from a pool of 18 consonants (all the consonants of the alphabet except $\mathrm{W}$ and $\mathrm{Z}$ ), avoiding obvious acronyms and alphabetically ordered strings. For each participant, every consonant appeared 20 times, four times at each position. Concerning the probes, there was an equal number of positive and negative probe trials. Each position in the sequence was probed an equal amount of times. For each participant and for all the 72 probes, every consonant was used four times, twice as positive probe and twice as negative.

A questionnaire (Difficulty Questionnaire) was used to assess the difficulty of picturing elements from right to left. Participants had to rate the difficulty using a scale from 1 to 5 ( 1 for "very easy", 2 for "quite easy", 3 for "average", 4 for "quite difficult" and 5 for "very difficult"). The aim of the questionnaire was to investigate whether the impression of difficulty could play a role in reversing the SPoARC effect. 


\subsection{Procedure}

Participants were tested one at a time in the presence of the experimenter. Earphones were used to deliver instructions. The experimental procedure had two phases: the training and the experiment phase.

In the training phase, participants had to complete a visual training to help them mentally visualize the memoranda in their mind from right to left. An item probe recognition task was used. ${ }^{14}$ The training was constituted by (four) sequences of five consonants to be remembered. All trials began with a "+" displayed, indicating that a sequence was going to be presented. Each consonant (size 72 in Courier New font, approximatively $1.9^{\circ}$ of visual angle) appeared successively from right to left on the screen (consonants were presented one at the time) at the rate of one letter every $3000 \mathrm{~ms}$. Each consonant, within a sequence, was separated from the previous or the following consonant by a distance of $8.9^{\circ}$ visual angle. Each consonant was also presented auditorily. Each sequence was followed by an interval of $2000 \mathrm{~ms}$ during which a sound indicated that a probe (a sixth consonant) would be presented. The probe was presented only auditorily. Participants were asked to indicate whether the probe was part of the sequence just heard by pressing a "yes" key or a "no" key. Half of the participants started this training phase using the left index finger to indicate "yes" by pressing "W" on a QWERTY keyboard (and used the right index finger to indicate "no" by pressing "1" on a QWERTY keyboard) and the other half of the participants started the training phase using the left index finger to indicate "no" by pressing "W" on a QWERTY keyboard (and used the right index finger to indicate "yes" by pressing “1” on a QWERTY keyboard). This left/right-hand key assignment was switched after half of the trials. Immediate auditory and visual feedback allowed participants to know whether they had succeeded the trial or not. 
In the experiment phase, participants were first blindfolded (as in Guida et al. ${ }^{13}$ ) to avoid any external visual stimulus while performing the experiment. Just afterwards they heard the first instruction (translated from French): "The following task is like the one you just finished except that the consonants to be remembered are going to be presented only auditorily. Moreover, we ask you to picture in your head each sequence of consonants from the right to the left. It is very important that you do so on each sequence until the end of the experiment". During the experiment phase, participants had to complete a familiarization block and a test block with one left/right-hand key assignment and subsequently a familiarization block and a test block with the converse left/right-hand key assignment. In addition to the first instruction (presented just above), before each familiarization or test block there was the following reminder (translated from French): "Don't forget to picture in your head each sequence of consonants from the right to the left". During each familiarization block, participants were familiarized with the item probe recognition task through four sequences of five consonants. Each familiarization block was followed by a test block that included 36 sequences of five consonants. All trials began with a tone display indicating that a sequence was going to be presented. Immediately after, five consonants were to be heard at a rate of $3000 \mathrm{~ms}$ per item. Each sequence was followed by a $2000 \mathrm{~ms}$ interval. During this interval, a sound would indicate that a probe (a consonant) was going to be presented. Once the probe was auditorily presented, participants had to judge whether or not the probe was part of the just-heard sequence, by pressing the "yes" key or the "no" key (keys W and 1) with the left or right index finger depending on the association requested. Just after their answer, a feedback indicated the accuracy of the answer. In the test blocks, the answer "yes" (and the answer "no") was assigned for the first 36 trials to one hand and for the 36 others to the other hand. This was counter-balanced across participants. The same 
hand was attributed to the "yes" answers in the familiarization block and in the test block that followed.

Finally, at the end of the experiment, participants took the Difficulty Questionnaire.

\section{Results}

We analyzed the data running the same statistical analyses than in Guida et al. ${ }^{13}$. Concerning accuracy, the overall score was $0.94(S D=0.23)$, and the average correct recognition score for “yes" answers was $0.94(S D=0.23)$. This result is almost identical to Guida et al. ${ }^{13}$ 's results (0.95 and 0.94 respectively). There was no speed accuracy trade-off: the correlation between RTs and errors over the 10 cells of the design was $r=0.39, p=0.27$.

Concerning reaction times (RT; raw data are available from OSF via the following link: https://osf.io/d9cp5/), the distribution skewness was dealt with by an inverse transform, as suggested by Brysbaert and Stevens, ${ }^{38}$ statistical analyses were performed by fitting linear mixed-effects model to inverse RTs, where both participants and items were entered as random effects. ${ }^{39}$ Trial number was included as a control variable to take any sequence effects in participants' RT (i.e. learning or fatigue effects) into account. For more information see Table 1 and its note.

Only correct recognitions for "yes" answers were analyzed, because "no" answers could not include the independent variable "Position in the sequence". The design was a $2 \times 5$ repeated measures with "Hand of response" for "yes" answers (left or right), and "Position in the sequence" that was probed (five positions) as within-subjects variables. The mean RT was 1833 ms $(S D=458)$.

Besides a significant positive effect of "Trial number" (participants' responses tended to take slightly longer in the last trials, across all conditions), a significant effect of "Hand of 
response" and "Position in the sequence" were observed (see Table 1 for the statistical results). The mean RT for the left hand was $1869 \mathrm{~ms}(S D=500)$ against $1797 \mathrm{~ms}(S D=408)$ for the right hand. For position 1 to 5 mean RTs were $1834 \mathrm{~ms}(S D=497), 1886 \mathrm{~ms}(S D=498), 1857 \mathrm{~ms}$ $(S D=453), 1824 \mathrm{~ms}(S D=458)$, and $1767 \mathrm{~ms}(S D=406)$. A significant interaction between "Hand of response" and "Position in the sequence" was also detected (Figure 1). A post-hoc power analysis was performed on this last effect, using the simr $\mathrm{R}$ package, ${ }^{40}$ which indicated a model comparison power of $73.30 \%$ (95\% CI [70.44, 76.02]).

It is to be noted that a left-handed participant was included in the sample. As left handers may perform differently on tasks involving lateral effects, we run the same analysis without the left-handed participant (see supplementary material, Table 1) a significant interaction between "Hand of response" and "Position in the sequence" was still observed.

$* * * * * * * * * * * * *$

(Table 1 \& Figure 1 about here)

$* * * * * * * * * * * * *$

In order to investigate the nature of the significant interaction with the standard procedure used in the SNARC and SPoARC domains, a regression analysis for repeated measures ${ }^{41}$ was applied to RTs differences (dRTs) obtained when subtracting the left hand RTs from the right hand RTs for each position and for each participant. ${ }^{42}$ To do so, RTs more than 2 standard deviations from the mean were excluded from the analysis: $4.5 \%$ of the data were removed. "Position in the sequence" was used as predictor and a one-tailed $t$ test was performed to evaluate if the regression weights of the group deviated significantly from zero. The test was significant, $t(29)=2.16, p=0.03$, dRTs increased by $23.9 \mathrm{~ms}$ per position (Figure 2 ).

Concerning this last analysis, it is to be noted that unlike all the other analyses we carried out, we 
entered "Participants" as a fixed factor, because usually $2,10,43$ this analysis is not done with random factors. However, if "Participants" is entered as a random factor, the $t$ is identical. Moreover, we run the same analysis without the left-handed participant, the $t$ remained almost unchanged, $t(28)=2.28, p=0.02$.

$* * * * * * * * * * * * *$

(Figure 2 about here)

$* * * * * * * * * * * * *$

\subsection{Comparison with Guida et al. ${ }^{13}$ 's results}

Figure 2 also exhibits results from Guida et al. ${ }^{13}$ who used the same procedure with the exceptions that participants were not asked to mentally picture the memoranda from right to left in their mind's eye. Compared to Guida et al. ${ }^{13}$, the SPoARC effect in the present study is reversed, which is an evidence of a right-to-left spatialization. In order to compare the magnitude of spatialization in both studies, we compared the absolute value of both slopes. For this purpose, we used the same method than in Guida et al. ${ }^{37}$, that is, we recoded the data from Guida et al. ${ }^{13}$ by reversing the "Position in the sequence" of dRTs (e.g., dRTs from position 1 were recoded as dRTs from position 5). Applying a Gaussian distribution to dRT through a linear mixed-effects model as in the above analysis, we compared a model that included "Participants" as random factor and "Position in the sequence" and "Experiment" (modalities: Guida et al. ${ }^{13}$ 's experiment vs. the present experiment) as fixed factors and we compare it to a model that included "Participants" as random factor and "Position in the sequence", "Experiment" and their interaction as fixed factors. The interaction did not lead to a significant improvement in fit, $\chi^{2}(1)$ $=0.13, \mathrm{p}=0.72$. Lastly, we used a Bayesian approach to test the difference between the two slopes. Again, there was a strong evidence (a BF10 inferior to 0.05 was found, which is 
considered strong evidence, see Ref. 44) in favor of the model that did not contain the interaction, which favors an absence of difference (in terms of magnitude) between the slopes in Guida et al. ${ }^{13}$ and in the present study (for the complete Bayesian analysis see supplementary material, Comparison with Guida et al. ${ }^{13}$ ).

\subsection{Analysis at an individual level}

We investigated the presence of the effect at the individual level using a bootstrapping technique put forward by Cipora et al. (Ref. 45, also see Ref. 46). It allows checking whether a given participant reveals the reliable spatialization effect, which cannot be attributed solely to measurement error. This allows investigating the prevalence of the effect under scrutiny. Here we used the H1 bootstrapping variant, which checks how the slope changes depending on which trials are included for aggregating. This method has recently been applied to the SPoARC effect by van Dijck et al. ${ }^{47}$ In this method all of the RTs of each participant for the different item position $\times$ hand combinations are randomly sampled (with replacement) and are used to calculate the slope. This is repeated 5000 times. Subsequently we established confidence intervals based on the range in which mid $80 \%$ of the bootstrap slopes were located (we used the same confidence level than van Dijck et al. ${ }^{47}$ to allow direct comparison with this study). In the last step, we checked whether such a confidence interval for a given participant contains zero (no evidence for reliable effect) or not (evidence for a reliable effect; see Ref. 45 for a detailed description of the method applied to SNARC data; the R scripts are available at https://osf.io/n7szg/). Among our 29 participants (this bootstrapping technique necessitates to drop participants that have no valid data in one condition i.e., item position $\times$ hand combinations, which was the case for one participant), $31 \%$ revealed reliable spatialization effect compared to $28 \%$ for the 29 participants in the Guida et al. ${ }^{13}$ 's experiment. Seven participants were found to 
spatialize from right to left in the present data (two from left to right) whereas eight participants were found to spatialize from left to right in the previous data (zero from right to left). We performed a Fisher exact test (suitable for a $2 \times 2$ contingency table with small samples) between the variable "Experiment" and a new variable stemming from the individual analysis named "Spatialization direction" (modalities: right-to-left spatializer vs. left-to-right spatializer) that lead to a significant $p=0.002$ value, allowing us to argue that we observed significant inversed patterns of spatialization between the two studies. For comparison, a bootstrapping analysis of Guida et al. ${ }^{36}$ 's results (testing right-to-left reading participants) run by van Dijck et al. ${ }^{47}$ showed that six (among 19) right-to-left reading participants exhibited a right-to-left SPoARC (two a left-to-right SPoARC). If one compares Guida et al. ${ }^{36}$ 's pattern (six right-to-left spatializers and two right-to-left spatializers) and the present data pattern (seven right-to-left spatializers and two right-to-left spatializers) using a Fisher exact test, the difference is nonsignificant.

\subsection{Questionnaire analysis}

Descriptive results were as follows: one participant rated the task as very difficult; four participants rated the task as quite difficult; seven participants rated the task as average; nine participants rated the task as quite easy; and seven participants rated the task as very easy. As above, we applied a Gaussian distribution to dRT through a linear mixed-effects model in order to compare a model that included "Participants" as random factor and "Position in the sequence" and "Difficulty" as fixed factors to a model that included "Participants" as random factor and "Position in the sequence", "Difficulty" and their interaction as fixed factors. The interaction only led to a marginal improvement in fit, $\chi^{2}(6)=3.44, p=0.06$. Although this result was nonsignificant, it is interesting to note that descriptively the trend showed that the more participants 
succeeded in reversing the SPoARC, the more they rated the task as difficult (see supplementary material, Figure 1).

\section{Discussion}

The aim of this study was to test whether the SPoARC effect could be reversed. Guida et al. ${ }^{36}$ have shown that right-to-left reading cultures have a right-to-left SPoARC effect whereas it is the converse for Westerners. Individuals seem therefore to spontaneously organize their thoughts on a horizontal line that follows the reading/writing direction system adopted in their educational system. The present study was designed to investigate the flexibility of this organization within Westerners. Can individuals reverse the cultural direction of their thoughts when asked to keep in mind a sequence?

For this purpose, participants were asked to remember 5-consonant sequences presented auditorily and were tested for a SPoARC effect. We used the experimental design of Guida et al. ${ }^{13}$ with the exception that participants were instructed to picture the memoranda from right to left. Compared to Guida et al. ${ }^{13}$, our results showed a reverse SPoARC. In addition, when comparing the SPoARC magnitude in both experiments via the slopes, we found no significant difference. In other words, spatialization seems as strong in the cultural direction (left to right in Guida et al. ${ }^{13}$ ) as in the non-cultural instructed direction (right to left in the present study). This extreme flexibility (no difference in terms slopes) however needs to be treated with caution as our participants were instructed to picture the memoranda from right to left, whereas in Guida et

al. ${ }^{13}$, participants received no instruction. It is possible that if we asked participants to picture the memoranda from left to right, the slope could be steeper than in Guida et al. ${ }^{13}$. Despite this caveat, the conclusion still stands, individuals have the flexibility to reverse the cultural direction of their thoughts. 


\subsection{Flexibility and working memory spatialization}

Our results resonate well with the mental whiteboard hypothesis, which proposes that space can be flexibly recruited to code order. Abrahamse et al. ${ }^{18}$ (p. 2) wrote: "the internal space that is used for serial order coding allows for flexibility: Coordinates along any well-arranged and orderly continuum (e.g., left to right; top to bottom; et cetera) may be recruited as best fits the task at hand." In our study the well-arranged spatial dimension was a right-to-left horizontal mental line. Therefore, although the internal (Western) default mechanism to code item sequences is from left to right, participants can use the reverse direction to code memoranda.

To analyze participants flexibility, we also investigated the presence of the effect at the individual level using bootstrapping. ${ }^{45} \mathrm{We}$ analyzed our data and Guida et al. ${ }^{13}$ 's results. The analysis revealed that in Guida et al. ${ }^{13}$ eight (among 29) participants showed a left-to-right SPoARC effect (none showed a right-to-left SPoARC). This pattern almost completely reversed in the present study where seven (among 29) participants showed a right-to-left SPoARC effect (two of them showed a left-to-right SPoARC). In addition, the pattern of the present study was close to that of Guida et al. ${ }^{36}$ where six (among 19) right-to-left reading participants exhibited a right-to-left SPoARC and two a left-to-right SPoARC (see the bootstrapping analysis run by van Dijck et al. ${ }^{47}$ ), despite the fact that both experimental set-ups were different (i.e., in Guida et al. ${ }^{36}$, participants had to memorize the color of four squares).

This kind of flexibility has also been observed by Guida et al. ${ }^{37}$ who showed that if sequences of consonants are presented to Western participants on a screen from right to left, participants do not re-spatialize the consonants from left to right but use the spatial information that is provided by the task at hand, and therefore encode the memoranda from right to left. 
The idea behind the flexibility of the cognitive system is related to a principle of economy that has been explicitly stated by Guida and Campitelli ${ }^{6}$ as the three steps of parsimony.

The first step states that if spatial information is already provided by the task at hand, the cognitive system will just use the spatial information in the environment (as in Guida et al. ${ }^{37}$ ). It is more economical for the cognitive system to use the spatial information already linked to memoranda than to re-spatialize the information (for other examples see the Simon effect ${ }^{48}$, or the visuospatial bootstrapping effect ${ }^{49-51}$ ), even if its direction is opposite to the reading direction. The kind of spatialization involved in this first step can be considered as a provided perceptual spatialization. It could be formed because attention is moved to a location (e.g., where a consonant is displayed on a screen), as proposed in the attention-shift hypothesis. ${ }^{52-55}$

Secondly, if the task does not provide spatial information in order to discriminate information to be processed or recalled (e.g., when items are presented one-by-one in the center of the screen), the cognitive system can use the spatial information that is related to the items in long-term memory (e.g., " 1 " is putatively on the left of " 2 " in semantic memory according to the mental numerical line ${ }^{2}$ ). This has been named long-term memory spatialization and can be observed in the SNARC effect. Third, if the cognitive system cannot use the latter two, it will spatialize information in WM on the fly, based on the direction of the reading/writing system (the first item entering WM will be linked to left). This constitute a WM spatialization.

SNARC and SPoARC were first thought to be explainable by WM spatialization (van Dijck and Fias ${ }^{10}$ named it the WM account) but following experiments ${ }^{11,31,32}$ showed that SNARC and SPoARC were distinct effects. The most convincing evidence is a study by Ginsburg and Gevers ${ }^{31}$ where they tweaked van Dijck and Fias ${ }^{10}$ original paradigm. In the 
original paradigm ${ }^{10}$ participants have to remember a sequence and then execute a go/no-go classification task (i.e., a parity judgment) on random numbers appearing on the screen. Crucially they are required to classify the numbers only if they belong to the memoranda. Ginsburg and Gevers ${ }^{31}$ made the following crucial change: they intermingled classic trials (like those in the original paradigm) with inducer trials where the classification task had to be executed for all numbers not only for those in the memoranda. Unlike van Dijck and Fias ${ }^{10}$ who just observed a SPoARC effect, Ginsburg and Gevers ${ }^{31}$ found both effects, demonstrating that SNARC and SPoARC could co-exist (for a similar result see Huber et al. ${ }^{32}$ ). Ginsburg et al. ${ }^{11}$ and Ginsburg and Gevers ${ }^{31}$ proposed that SNARC and SPOARC derive from two different processes, the former based on long-term memory semantic knowledge (with numbers ordered canonically, here named long-term memory spatialization) and the latter based on the order items enter WM (here named WM spatialization). The dual nature of the effects was the reason why both effects could be detected conjunctly.

Up to now, we knew that perceptual spatialization could be reversed (see Guida et al. ${ }^{37}$ presented above). We also knew that long-term memory spatialization could be reversed by directly asking Western participants to think about a right to left numerical mental line while performing a SNARC task ${ }^{34}$ (a top-down control reversion). It could also be reversed indirectly as in Fischer et al. ${ }^{56}$, where they asked participants (a group of Hebrew students and a group of Scottish students) to read (during 20 minutes) cook recipes in which small and large numbers appeared near the left or right ends of the lines. These numbers were positioned congruently to one's reading direction or incongruently. A subsequent SNARC test showed that those 20 minutes of reading where sufficient in the incongruent condition to reverse the SNARC effect in the Hebrew group and to reduce it in the Scottish group (for a review see Ref. 35). 
However, up to now, nobody knew if WM spatialization could be flexibly reversed. We now know that it is also the case for WM spatialization.

\subsection{Flexibility and memory performance}

Although the cognitive system seems very flexible in terms of spatialization, encoding information not congruently to one's reading/writing direction seems to hinder memory performance. It was not the case in the present study as our results (with a right-to-left encoding) in terms of accuracy are almost identical to Guida et al. ${ }^{13}$ 's results (with a left-to-right encoding). We believe the cause to be a ceiling effect, as SNARC tasks or SPoARC tasks are designed to be easy because the principal dependent variable is RT. In tasks that are not designed to be easy, performance (when information is not congruently to one's reading/writing direction) can be hampered. For example, Fischer-Baum and Benjamin ${ }^{57}$ asked their participants to remember sequences of 6 letters, which could appear from left to right or right to left. The former condition produced better results, regardless if participants were asked to perform a backward or forward recall. Similarly, McCrink and Shaki ${ }^{58}$ asked American participants (who write words from left to right) and Israeli participants (who write words from right to left) to learn seven pairs constituted by one letter and one geometrical figure (McCrink \& Shaki ${ }^{58}$, Experiment 1). The pairs were displayed either centrally, from left to right, or from right to left. Learning performance was higher when the presentation of the pairs at encoding matched the directionality of the reading/writing system. Overall, there seemed to be a mnemonic cost for processing memoranda incongruently to one's reading/writing direction, even though participants can reverse they SPoARC on demand.

One possible explanation for this discrepancy could be that although individuals can spatially encode memoranda from right to left, it comes at a price in terms of cognitive resources. 
Our results from the Difficulty Questionnaire hint at that. The trend (the statistical result was only marginal) shows that participants who were able to reverse the SPoARC found the task more difficult than those who failed to reverse the SPoARC (see supplementary material, Figure 1). This could be related to the recent theoretical framework put forward by Guida and Campitelli. ${ }^{6}$ They proposed that spatialization is used by the cognitive system to organize and stabilize thoughts in WM. Usually the capacity to stabilize thoughts in WM in face of interference is linked to attentional control (or cognitive control, executive control, central executive, controlled attention, executive functioning, depending on the theory ${ }^{59-62}$ ) but for Guida and Campitelli ${ }^{6}$ another important element are spatial schemas, because these allow to reduce our need for attentional control. Although literate adults spontaneously use a horizontal spatial schema, various spatial schemas can be used such as locations in the loci method. Guida and Campitelli ${ }^{6}$ propose, as in Norman and Shallice ${ }^{63}$ 's supervisory attentional system, that learned spatial schemas (or schemata in Norman and Shallice's theory) enable using less attention, as it is only when appropriate long-term memory schemas cannot be used that attentional control must intervene heavily. We believe that a similar process occurred in the present experiment: when participants were asked to encode the memoranda from right to left, they could not use the practiced left-to-right spatial schema. To execute the task properly, our interpretation is that they were constraint to rely more on attentional control. This could explain why participants who managed to reverse the SPoARC effect found the task more difficult. As this rationale is based only on a marginal effect, it must be taken with caution.

\subsection{Spatialization as a means to code order}

Finally, we believe that our results and more generally the SPoARC effect can contribute to the understanding of how order is coded in memory. Since the seventies, results (e.g., Refs. 
64-70) have shown that memory models have to account for two kinds of information: memory for the content and memory for the order. Item and order information seem to be coded and represented separately (for reviews, see Refs. 23, 24, 71). Consequentially various theories have assumed that item information and order information were coded separately, although, up to now, it remains unclear how exactly order is coded in memory. Several very different mechanisms are still in competition and different classifications (that sometimes do not overlap) have been proposed. For Henson ${ }^{72}$ for example, memory models can be classified in three categories -chaining, ordinal, or positional models-and additionally the position of the items can be coded in temporal, absolute, or relative terms. Marshuetz ${ }^{24}$ instead, has proposed five classes of mechanisms: inter-item association, direct coding, hierarchical coding, feature coding, and magnitude coding. Lewandowsky and Farrell ${ }^{73}$ have proposed four different types of items associations to code order: item-context (e.g., Ref. 74), item-temporal context (e.g., Ref. 28) item-item (e.g., Ref. 26) and recurrent associations (e.g., Ref. 75). We will use their classification.

If the first prominent idea (Ebbinghaus ${ }^{76}$ ) to explain how order was coded was based on item-item associations, in the last two decades item-context models (as well as item-temporal context models, but in a lesser degree because these models have been less disseminated and are still empirically uncertain ${ }^{77-79}$ ) have gained ground. In these models, order is coded through positional coding mechanisms, where a positional marker/tag (the context) is associated to each item. This mechanism is found in models accounting for short-term memory, WM and episodic memory (e.g., Refs. 27,28,73,80-84).

The main problem of item-context models is that they do not explicitly describe the nature of the positional markers (see Ref. 73 for an explicit statement). Interestingly, what 
transpires from the research on the SPoARC effect is that order coding could be spatial, which could help give flesh to the positional markers revealing their spatial nature.

More specifically, our results confirm the direct nature of these spatial codes. Recently, Guida et al. ${ }^{37}$ proposed that space could be used as positional markers in at least two ways (a direct or an indirect way). In the direct hypothesis, when a to-be-remembered sequence (e.g., "G", "D", "B") enters WM, it directly receives a spatial positional marker in terms of context: as "G" is the first to enter WM it acquires a relatively (i.e., compared to the other items) left spatial value (in western cultures) as positional marker, "D" acquires a relatively middle spatial value and " $\mathrm{B}$ " a relatively right spatial value. In the indirect hypothesis, when the items of a sequence enter WM, they do not get linked to space directly. Instead they are first linked to a positional marker, which can be a number ("G" is linked to 1 , " $\mathrm{D}$ " to 2 , and "B" to 3 ) or a rank ("G" is linked to $1^{\text {st }}$, "D" to $2^{\text {nd }}$, and "B" to $3^{\text {rd }}$ ). Young ${ }^{85}$ and Ebenholtz ${ }^{86}$ were among the firsts to propose these kinds of positional markers, which have re-emerged since (e.g., Refs. 87-91). As far as the indirect hypothesis is concerned, these numbers or ranks are subsequently transformed into space (for more details see Guida et al. ${ }^{37}$ ) thanks to the long-term memory knowledge individuals possess of the association between numbers and space (as proposed by Dehaene et al. ${ }^{2}$ 's semantic hypothesis): as 1 or $1^{\text {st }}$ are (relatively to 2 or $2^{\text {nd }}$ ) associated to left " $G$ " acquires a left spatial value, " $D$ " via 2 or $2^{\text {nd }}$ acquires a relatively middle spatial value and " $B$ " via 3 or $3^{\text {rd }}$ a relatively right spatial value. Both hypotheses can account for the SPoARC effect.

One way to pitch the direct hypothesis against the indirect hypothesis is to display items on the screen from right to left in a SPoARC task like Guida et al. ${ }^{37}$ did. In the direct tagging hypothesis and following the mental whiteboard hypothesis, ${ }^{18}$ participants should directly use the available spatial information on the screen (the spatial coordinates of the items) to derive 
positional markers to code order, resulting in a right-to-left representation. Conversely, if the indirect spatial tagging hypothesis is correct, a left-to-right representation should be observed. Going back to our example, the rationale is as follows. If participants see the sequence "G", "D", "B" displayed from right to left, they should associate the first item ("G") with the marker 1 or $1^{\text {st }}$, the second item ("D") with 2 or $2^{\text {nd }}$, and the third item ("B") with 3 or $3^{\text {rd }}$. And according to the indirect hypothesis, "G" via 1 or $1^{\text {st }}$ should then be linked to a left marker, "D" via 2 or $2^{\text {nd }}$ to a middle marker and "B" via 3 or $3^{\text {rd }}$ to a right marker, generating a left-to-right SPoARC. Results from Guida et al. ${ }^{37}$ favored the direct hypothesis.

Our experimental design is another way to test the indirect hypothesis. Given that the participants are asked to picture in their minds items from right to left, a left-to-right SPoARC should emerge according to the indirect hypothesis, exactly as in the example above. The only difference being that in Guida et al. ${ }^{37}$ 's paradigm, items were displayed (externally) on the screen from right to left, whereas in the present study, participants have to generate themselves, internally, the spatial coordinates of the items. Our study shows that this element does not change the outcome, results show a right-to-left SPoARC effect which is not compatible with the indirect hypothesis put forward by Guida et al. ${ }^{37}$

To conclude, with this study we have shown that when individuals are asked to keep in mind a random sequence, the SPoARC effect can be reversed. This provides some clues on how our thoughts can be organized. Although culture clearly shapes the way we organize our thoughts, ${ }^{36}$ our result shows that we are nonetheless able to reverse them, with the caveat provided by the literature that this could be detrimental in terms of cognitive resources and mnemonic performance. Future research will certainly need to clarify this point. 


\section{References}

1. Guida, A., Carnet, S., Normandon, M. \& Lavielle-Guida, M. Can spatialisation be extended to episodic memory and open sets? Memory 26, 922-935 (2018).

2. Dehaene, S., Bossini, S. \& Giraux, P. The mental representation of parity and number magnitude. Journal of Experimental Psychology: General 122, 371 (1993).

3. Bonato, M., Zorzi, M. \& Umiltà, C. When time is space: Evidence for a mental time line. Neuroscience \& Biobehavioral Reviews 36, 2257-2273 (2012).

4. Abrahamse, E., van Dijck, J.-P. \& Fias, W. How does working memory enable numberinduced spatial biases? Frontiers in Psychology 7, (2016).

5. Gevers, W. \& Lammertyn, J. The hunt for SNARC. Psychology Science 47, 10-21 (2005).

6. Guida, A. \& Campitelli, G. Explaining the SPoARC and SNARC effects with knowledge structures: An expertise account. Psychonomic bulletin \& review 26, 434-451 (2019).

7. Wood, G., Willmes, K., Nuerk, H.-C. \& Fischer, M. H. On the cognitive link between space and number: A meta-analysis of the SNARC effect. Psychology Science Quarterly 50, 489 (2008).

8. Shaki, S., Fischer, M. H. \& Petrusic, W. M. Reading habits for both words and numbers contribute to the SNARC effect. Psychonomic Bulletin \& Review 16, 328-331 (2009).

9. Zebian, S. Linkages between number concepts, spatial thinking, and directionality of writing: The SNARC effect and the reverse SNARC effect in English and Arabic monoliterates, biliterates, and illiterate Arabic speakers. Journal of Cognition and Culture 5, 1-2 (2005). 
10. van Dijck, J.-P. \& Fias, W. A working memory account for spatial-numerical associations. Cognition 119, 114-119 (2011).

11. Ginsburg, V., van Dijck, J.-P., Previtali, P., Fias, W. \& Gevers, W. The impact of verbal working memory on number-space associations. Journal of Experimental Psychology: Learning, Memory, and Cognition 40, 976 (2014).

12. Becker, S. L. The ordinal position effect. Quarterly Journal of Speech 39, 217-219 (1953).

13. Guida, A., Leroux, A., Lavielle-Guida, M. \& Noël, Y. A SPoARC in the dark: Spatialization in verbal immediate memory. Cognitive Science 40, 2108-2121 (2016).

14. Sternberg, S. High-speed scanning in human memory. Science 153, 652-654 (1966).

15. Antoine, S., Ranzini, M., Gebuis, T., van Dijck, J.-P. \& Gevers, W. Order information in verbal working memory shifts the subjective midpoint in both the line bisection and the landmark tasks. The Quarterly Journal of Experimental Psychology 70, 1973-1983 (2017).

16. Ginsburg, V., Archambeau, K., van Dijck, J.-P., Chetail, F. \& Gevers, W. Coding of serial order in verbal, visual and spatial working memory. Journal of Experimental Psychology: General 146, 632 (2017).

17. Rinaldi, L., Brugger, P., Bockisch, C. J., Bertolini, G. \& Girelli, L. Keeping an eye on serial order: Ocular movements bind space and time. Cognition 142, 291-298 (2015).

18. Abrahamse, E., van Dijck, J.-P., Majerus, S. \& Fias, W. Finding the answer in space: the mental whiteboard hypothesis on serial order in working memory. Front Hum Neurosci 8, (2014). 
19. Abrahamse, E. L., van Dijck, J.-P. \& Fias, W. Grounding verbal working memory: The case of serial order. Current Directions in Psychological Science 26, 429-433 (2017).

20. Guida, A. \& Lavielle-Guida, M. 2011 space odyssey: spatialization as a mechanism to code order allows a close encounter between memory expertise and classic immediate memory studies. Psychological perspectives on expertise 5, 176 (2014).

21. Previtali, P., de Hevia, M. D. \& Girelli, L. Placing order in space: the SNARC effect in serial learning. Experimental Brain Research 201, 599-605 (2010).

22. Bonato, M., Saj, A. \& Vuilleumier, P. Hemispatial neglect shows that "before" is "left". Neural plasticity 2016, (2016).

23. Hurlstone, M. J., Hitch, G. J. \& Baddeley, A. D. Memory for serial order across domains: An overview of the literature and directions for future research. Psychological bulletin 140, 339 (2014).

24. Marshuetz, C. Order information in working memory: an integrative review of evidence from brain and behavior. Psychological bulletin 131, 323 (2005).

25. Henson, R. N. Short-term memory for serial order: the Start-End Model. Cognitive psychology 36, 73-137 (1998).

26. Lewandowsky, S., Murdock, B. B. \& others. Memory for serial order. Psychological Review 96, 25-57 (1989).

27. Brown, G. D. A., Neath, I. \& Chater, N. A temporal ratio model of memory. Psychological review 114, 539-76 (2007).

28. Brown, G. D., Preece, T. \& Hulme, C. Oscillator-based memory for serial order. Psychological review 107, 127 (2000). 
29. Patro, K., Nuerk, H.-C., Cress, U. \& Haman, M. How number-space relationships are assessed before formal schooling: A taxonomy proposal. Frontiers in Psychology $\mathbf{5}$, (2014).

30. McCrink, K. \& de Hevia, M. D. From innate spatial biases to enculturated spatial cognition: The case of spatial associations in number and other sequences. Frontiers in psychology 9, 415 (2018).

31. Ginsburg, V. \& Gevers, W. Spatial coding of ordinal information in short-and long-term memory. Frontiers in human neuroscience 9, (2015).

32. Huber, S., Klein, E., Moeller, K. \& Willmes, K. Spatial-numerical and ordinal positional associations coexist in parallel. Frontiers in psychology 7, (2016).

33. Bächtold, D., Baumüller, M. \& Brugger, P. Stimulus-response compatibility in representational space. Neuropsychologia 36, 731-735 (1998).

34. Ristic, J., Wright, A. \& Kingstone, A. The number line effect reflects top-down control. Psychonomic bulletin \& review 13, 862-868 (2006).

35. Cipora, K., Patro, K. \& Nuerk, H.-C. Situated influences on spatial-numerical associations. In Spatial Biases in Perception and Cognition. T. Hubbard, Ed.: 41-59. Cambridge University Press (2018).

36. Guida, A. et al. Spatialization in working memory is related to literacy and reading direction: Culture "literarily" directs our thoughts. Cognition 175, 96-100 (2018).

37. Guida, A., Abrahamse, E. \& van Dijck, J.-P. About the interplay between internal and external spatial codes in the mind: implications for serial order. Annals of the New York Academy of Sciences (2020). 
38. Brysbaert, M. \& Stevens, M. Power analysis and effect size in mixed effects models: A tutorial. Journal of cognition 1, (2018).

39. Baayen, R. H., Davidson, D. J. \& Bates, D. M. Mixed-effects modeling with crossed random effects for subjects and items. Journal of memory and language 59, 390-412 (2008).

40. Green, P. \& MacLeod, C. J. SIMR: an R package for power analysis of generalized linear mixed models by simulation. Methods in Ecology and Evolution 7, 493-498 (2016).

41. Lorch, R. F. \& Myers, J. L. Regression analyses of repeated measures data in cognitive research. Journal of Experimental Psychology: Learning, Memory, and Cognition 16, 149 (1990).

42. Fias, W., Brysbaert, M., Geypens, F. \& d'Y dewalle, G. The importance of magnitude information in numerical processing: Evidence from the SNARC effect. Mathematical Cognition 2, 95-110 (1996).

43. Fias, W. The importance of magnitude information in numerical processing: Evidence from the SNARC effect. Mathematical cognition 2, 95-110 (1996).

44. Raftery, A. E. Bayesian model selection in social research. Sociological methodology 25, 111-164 (1995).

45. Cipora, K. et al. A Minority pulls the sample mean: on the individual prevalence of robust group-level cognitive phenomena-the instance of the SNARC effect. (2019).

46. Hohol, M. et al. Professional mathematicians do not differ from others in the symbolic numerical distance and size effects. Scientific reports 10, 1-12 (2020). 
47. van Dijck, J.-P., Abrahamse, E., Fias, W. \& van Dijck, J.-P. Do preliterate children spontaneously employ spatial coding for serial order in working memory? Annals of the New York Academy of Sciences (2020).

48. Simon, J. R. \& Rudell, A. P. Auditory SR compatibility: the effect of an irrelevant cue on information processing. Journal of applied psychology 51, 300 (1967).

49. Allen, R. J., Havelka, J., Falcon, T., Evans, S. \& Darling, S. Modality specificity and integration in working memory: Insights from visuospatial bootstrapping. Journal of Experimental Psychology: Learning, Memory, and Cognition 41, 820 (2015).

50. Darling, S., Allen, R. J., Havelka, J., Campbell, A. \& Rattray, E. Visuospatial bootstrapping: Long-term memory representations are necessary for implicit binding of verbal and visuospatial working memory. Psychonomic Bulletin \& Review 19, 258-263 (2012).

51. Darling, S. \& Havelka, J. Visuospatial bootstrapping: Evidence for binding of verbal and spatial information in working memory. The Quarterly Journal of Experimental Psychology 63, 239-245 (2010).

52. Stoffer, T. H. Attentional focussing and spatial stimulus-response compatibility. Psychological research 53, 127-135 (1991).

53. Stoffer, T. H. \& Umiltà, C. Spatial coding with reference to the focus of attention in SRcompatibility and the Simon effect. in b. hommel \& w. prinz (eds.), Theoretical issues inS-R compatibility (pp. 181-208). (Amsterdam: North-Holland, 1997).

54. Umiltà, C. \& Nicoletti, R. An integrated model of the Simon effect. (1992).

55. Umilta, C., Bonato, M. \& Rusconi, E. S-R compatibility with physical and representational locations. Spatial biases in perception and cognition 60-76 (2018). 
56. Fischer, M. H., Mills, R. A. \& Shaki, S. How to cook a SNARC: Number placement in text rapidly changes spatial-numerical associations. Brain and Cognition 72, 333-336 (2010).

57. Fischer-Baum, S. \& Benjamin, A. S. Time, space, and memory for order. Psychonomic bulletin \& review 21, 1263-1271 (2014).

58. McCrink, K. \& Shaki, S. Culturally inconsistent spatial structure reduces learning. Acta psychologica 169, 20-26 (2016).

59. Chow, M. \& Conway, A. R. The scope and control of attention: Sources of variance in working memory capacity. Memory \& cognition 43, 325-339 (2015).

60. Engle, R. W. Working memory capacity as executive attention. Current directions in psychological science 11, 19-23 (2002).

61. Kane, M. J., Bleckley, M. K., Conway, A. R. \& Engle, R. W. A controlled-attention view of working-memory capacity. Journal of Experimental Psychology: General 130, 169 (2001).

62. Kane, M. J. \& Engle, R. W. Working-memory capacity and the control of attention: the contributions of goal neglect, response competition, and task set to Stroop interference. Journal of experimental psychology: General 132, 47 (2003).

63. Norman, D. A. \& Shallice, T. Attention to action. in Consciousness and self-regulation 1-18 (Springer, 1986).

64. Bjork, R. A. \& Whitten, W. B. Recency-sensitive retrieval processes in long-term free recall. Cognitive Psychology 6, 173-189 (1974).

65. Dale, R. H. Similarities between human and animal spatial memory: Item and order information. Animal Learning \& Behavior 15, 293-300 (1987). 
66. Engelkamp, J. \& Dehn, D. M. Item and order information in subject-performed tasks and experimenter-performed tasks. Journal of Experimental Psychology: Learning, Memory, and Cognition 26, 671 (2000).

67. Henson, R. N. Unchained memory: Error patterns rule out chaining models of immediate serial recall. The Quarterly Journal of Experimental Psychology: Section A 49, 80-115 (1996).

68. Mulligan, N. W. The effects of perceptual interference at encoding on organization and order: Investigating the roles of item-specific and relational information. Journal of Experimental Psychology: Learning, Memory, and Cognition 25, 54 (1999).

69. Majerus, S., Poncelet, M., Elsen, B. \& van der Linden, M. Exploring the relationship between new word learning and short-term memory for serial order recall, item recall, and item recognition. European Journal of Cognitive Psychology 18, 848-873 (2006).

70. Sperling, G. \& Melchner, M. J. Estimating item and order information. Journal of Mathematical Psychology 13, 192-213 (1976).

71. Majerus, S. \& Attout, L. Working memory for serial order and numerical cognition: What kind of association? in Heterogeneity of function in numerical cognition 409-431 (Elsevier, 2018).

72. Henson, R. N. Coding position in short-term memory. International Journal of Psychology 34, 403-409 (1999).

73. Lewandowsky, S. \& Farrell, S. Short-term memory: New data and a model. Psychology of Learning and Motivation 49, 1-48 (2008). 
74. Portrat, S., Guida, A., Phénix, T. \& Lemaire, B. Promoting the experimental dialogue between working memory and chunking: Behavioral data and simulation. Memory \& cognition 44, 420-434 (2016).

75. Botvinick, M. M. \& Plaut, D. C. Empirical and computational support for contextdependent representations of serial order: Reply to Bowers, Damian, and Davis (2009). Psychological Review 116, 998-1001 (2009).

76. Ebbinghaus, H. Ueber das Gedächtnis. (1885).

77. Brown, G. D. \& Lewandowsky, S. Serial recall and presentation schedule: A microanalysis of local distinctiveness. Memory 13, 283-292 (2005).

78. Lewandowsky, S., Duncan, M. \& Brown, G. D. Time does not cause forgetting in shortterm serial recall. Psychonomic Bulletin \& Review 11, 771-790 (2004).

79. Lewandowsky, S., Brown, G. D. A., Wright, T. \& Nimmo, L. M. Timeless memory: Evidence against temporal distinctiveness models of short-term memory for serial order. Journal of Memory and Language 54, 20-38 (2006).

80. Oberauer, K., Lewandowsky, S., Farrell, S., Jarrold, C. \& Greaves, M. Modeling working memory: An interference model of complex span. Psychonomic Bulletin \& Review 19, 779-819 (2012).

81. Anderson, J. R. \& Matessa, M. A production system theory of serial memory. Psychological Review 104, 728 (1997).

82. Davelaar, E. J., Goshen-Gottstein, Y., Ashkenazi, A., Haarmann, H. J. \& Usher, M. The demise of short-term memory revisited: empirical and computational investigations of recency effects. Psychological review 112, 3-42 (2005). 
83. Polyn, S. M., Norman, K. A. \& Kahana, M. J. A context maintenance and retrieval model of organizational processes in free recall. Psychological review 116, 129-56 (2009).

84. Sederberg, P. B., Howard, M. W. \& Kahana, M. J. A context-based theory of recency and contiguity in free recall. Psychological review 115, 893-912 (2008).

85. Young, R. K. Tests of three hypotheses about the effective stimulus in serial learning. Journal of Experimental Psychology 63, 307 (1962).

86. Ebenholtz, S. M. Serial learning: Position learning and sequential associations. Journal of Experimental Psychology 66, 353 (1963).

87. Burgess, C., Schuck, N. W. \& Burgess, N. Temporal Neuronal Oscillations can Produce Spatial Phase Codes. in Space, Time and Number in the Brain 59-69 (Elsevier, 2011).

88. Botvinick, M. \& Watanabe, T. From Numerosity to Ordinal Rank: A Gain-Field Model of Serial Order Representation in Cortical Working Memory. Journal of Neuroscience 27, 8636-8642 (2007).

89. Müller, D. \& Schwarz, W. "1-2-3”: Is There a Temporal Number Line?: Evidence from a Serial Comparison Task. Experimental Psychology (formerly 'Zeitschrift für Experimentelle Psychologie') 55, 143-150 (2008).

90. Nieder, A. Counting on neurons: the neurobiology of numerical competence. Nature Reviews Neuroscience 6, 177-190 (2005).

91. Schwarz, W. \& Eiselt, A.-K. The perception of temporal order along the mental number line. Journal of Experimental Psychology: Human perception and performance 35, 989 (2009). 
92. Noel, Y. R2STATS: A GTK GUI for GLM and GLMM in R (R package version 0.6829). R package version 0.68-29) [Software]. Retrieved from http:// cran.rproject.org/package $=R 2 S T A T S$.

93. Bates, D. M. Bates, D., Maechler, M., \& Bolker, B. (2011). lme4: Linear mixedeffects models using S4 classes (R package version 0.999375-42) [Software]. Retrieved from http://CRAN.R-project.org/package= lme4.

94. R Development Core Team. (2012). R: A language and environment for statistical computing [Computer software]. Available at: http://www.R-project.org. Accessed June 4, 2015.

95. Schwarz, G. Estimating the dimension of a model. The annals of statistics 6, 461-464 (1978).

96. Jeffreys, H. Theory of probability, Clarendon. (Oxford, 1961).

97. Wagenmakers, E.-J. A practical solution to the pervasive problems ofp values. Psychonomic bulletin \& review 14, 779-804 (2007). 


\section{Conflict of Interest Statement}

The authors declare that the research was conducted in the absence of any commercial or financial relationships that could be construed as a potential conflict of interest. 
Table 1. Results of linear mixed-effects model comparison comprising two independent variables: "Position in the sequence", "Hand of response."

\begin{tabular}{cccccc}
\hline Model & Effect & Deviance & $\chi^{2}$ & $d f$ & $P$ \\
\hline 0 & & 2748.6 & & & \\
1 & Trial number & 2741.2 & 7.42 & 1 & 0.006 \\
2 & Hand & 2727.5 & 13.73 & 1 & 0.0002 \\
3 & Position & 2720.9 & 6.50 & 1 & 0.011 \\
4 & Position $\times$ Hand & 2714.6 & 6.32 & 1 & 0.012 \\
\hline
\end{tabular}

Note: All models included participants as random effects. "Hand" stands for "Hand of response" and "Position" for "Position in the sequence". Models were fitted using a restricted maximum likelihood technique. The R2STATS ${ }^{92}$ package was used, which is based on the lme4 package ${ }^{93}$ in $R$ language.$^{94}$ Initially, a model that only included random factors (items and participants) was applied to the data (Model 0). This original model was then enriched by sequentially adding the control variable (Trial number), and then the two predictors, Hand and Position (taken as numeric) and their interaction. The improvement of fit of each model was statistically tested using a Likelihood-Ratio Chi-Square test. The procedure is very similar to testing for the significance of increments to $R^{2}$ resulting from the addition of explanatory variables to a multiple regression equation. 


\section{Figure captions}

Figure 1. Distribution of mean observed data and linear fit for left-hand reaction times and right-hand reaction times as a function of the probed position in the sequence.

Figure 2. Comparison of the data from the present study and from Guida et al. ${ }^{13}$. In black, mean observed data from the present study and linear fit for right-hand reaction times minus left-hand reaction times as a

function of the probed position in the sequence. In red, mean observed data from Guida et al. ${ }^{13}$ and linear fit representing right-hand reaction times minus left-hand reaction times as a function of the probed position in the sequence. 
Figure 1
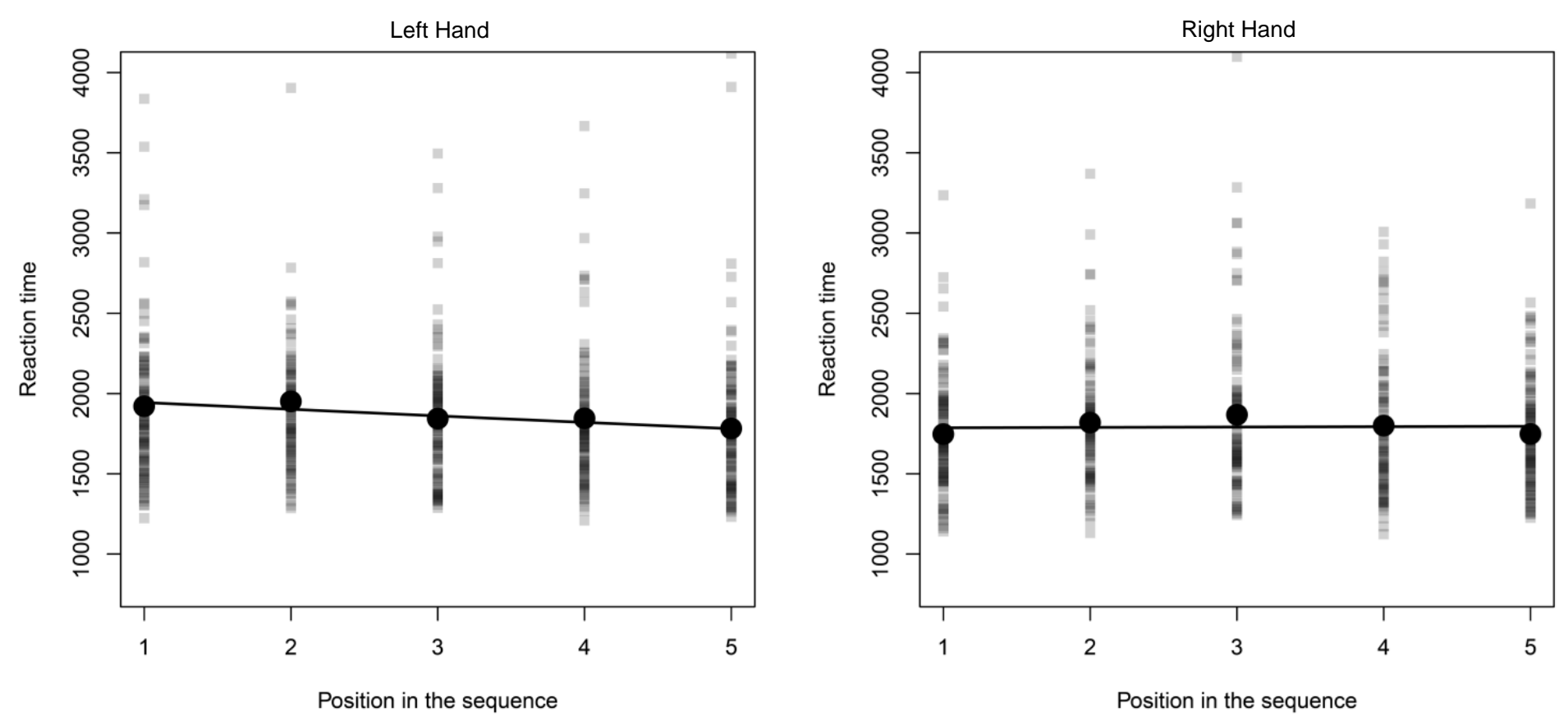
Figure 2

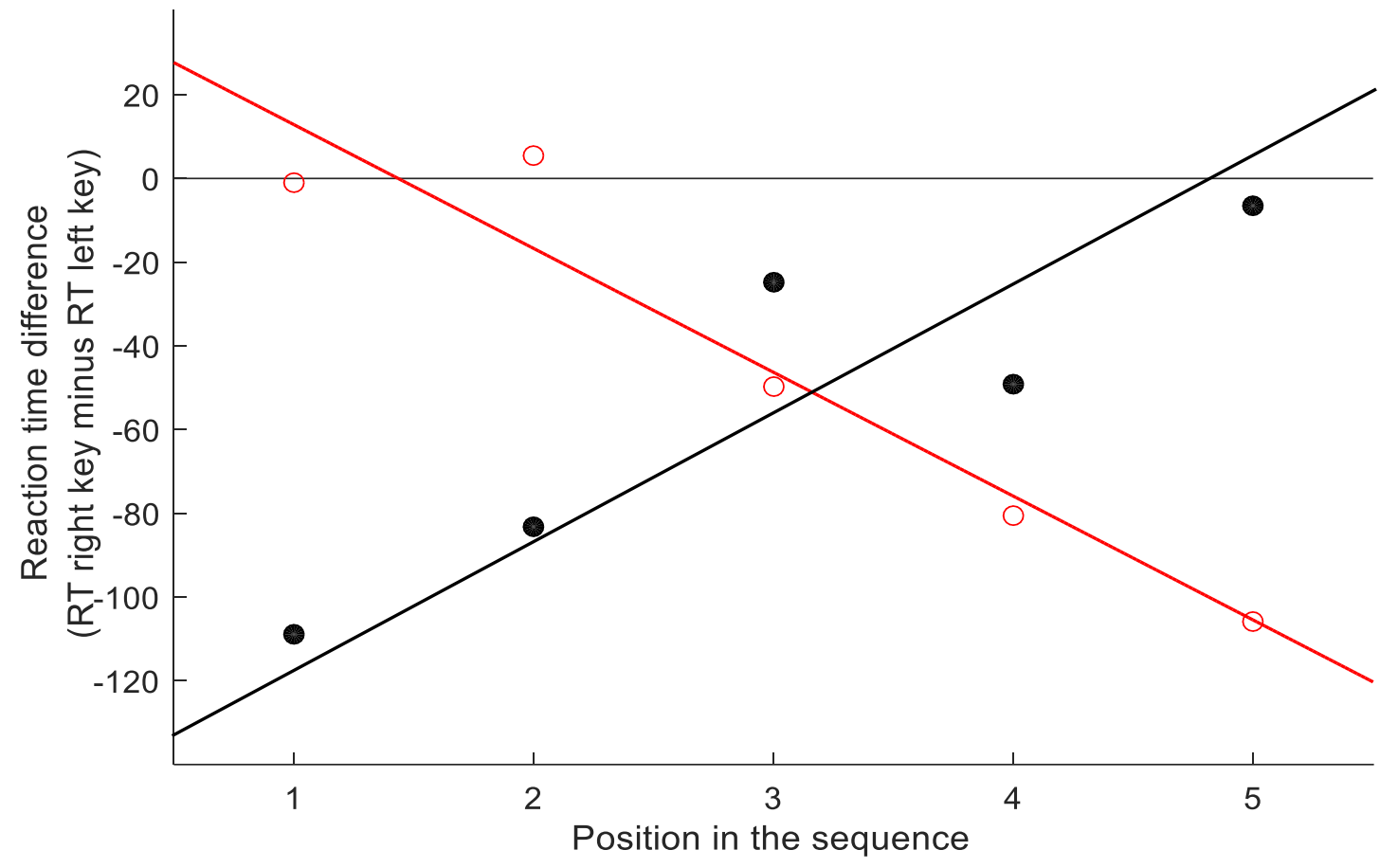




\section{Supplementary Material}

Table 1. Results of a linear mixed-effects model without the left-handed participant, comparison comprising two independent variables: "Position in the sequence", "Hand of response."

\begin{tabular}{cccccc}
\hline Model & Effect & Deviance & $\chi^{2}$ & $d f$ & $P$ \\
\hline 0 & & 2572.0 & & & \\
1 & Trial number & 2566.1 & 5.91 & 1 & 0.02 \\
2 & Hand & 2556.7 & 9.37 & 1 & 0.002 \\
3 & Position & 2551.9 & 4.81 & 1 & 0.03 \\
4 & Position $\times$ Hand & 2547.7 & 4.20 & 1 & 0.04 \\
\hline
\end{tabular}




\section{Comparison with Guida et al. ${ }^{13}$}

Two models were compared, a model (the non-interaction model) that included "Participants" as random factor and "Position in the sequence" and "Experiment" (modalities: Guida et al. ${ }^{13}$ 's experiment vs. the present experiment) as fixed factors and a model (the interaction model) that included "Participants" as random factor and "Position in the sequence", "Experiment" and their

interaction as fixed factors. We used the BIC (Bayesian Information Criterion ${ }^{95}$ ) of the two models to compute a Bayes factor ${ }^{96}$ which allows to compare the two models. We first computed the BIC for the interaction model $\left(H_{1}\right)$ and the non-interaction model $\left(H_{0}\right)$ which revealed a $\mathrm{BIC}\left(H_{1}\right)$ of 4132 versus a $\mathrm{BIC}\left(H_{0}\right)$ of 4126 . Then via the $\mathrm{BICs}$ difference, the Bayes factor $\mathrm{BF}_{10}$ was computed using the formula (also see Ref. ${ }^{97}$ ) as follows

$$
\mathrm{BF}_{10}=e^{\left(\frac{\Delta B I C 10}{2}\right)}
$$

We found a $\mathrm{BF}_{10}$ that was just inferior to 0.05 . Based on Raftery ${ }^{44}$ this is strong evidence in favor $H_{0}$. In other words, there is a strong evidence against an interaction between "Position in the sequence" and "Experiment", which is in favor of an absence of difference in terms of magnitude between the slopes of the present study and Guida et al. ${ }^{13}$ 's one. To be complete these are the interpretation of $\mathrm{BF}_{10}$ in terms of evidence ${ }^{44}$ : a $\mathrm{BF}_{10}$ inferior to $1 / 150(0.007)$ is a very strong evidence in favor of $\mathrm{M}_{0} ; 1 / 150(0.007)<\mathrm{BF}_{10}<1 / 20(0.05)$ is a strong evidence in favor of $\mathrm{M}_{0} ; 1 / 20(0.05)<\mathrm{BF}_{10}<1 / 3(0.33)$ is a positive evidence in favor of $\mathrm{M}_{0} ; 1 / 3(0.33)<\mathrm{BF}_{10}<1$ is a weak evidence in favor of $\mathrm{M}_{0}$. 


\section{Figure captions}

Figure 1. Linear fit for right-hand reaction times minus left-hand reaction times as a function of the probed position in the sequence for each group that was constituted based on their answer to the Difficulty Questionnaire ( $\mathrm{N}$ indicates the number of participants in each group) 
Figure 1

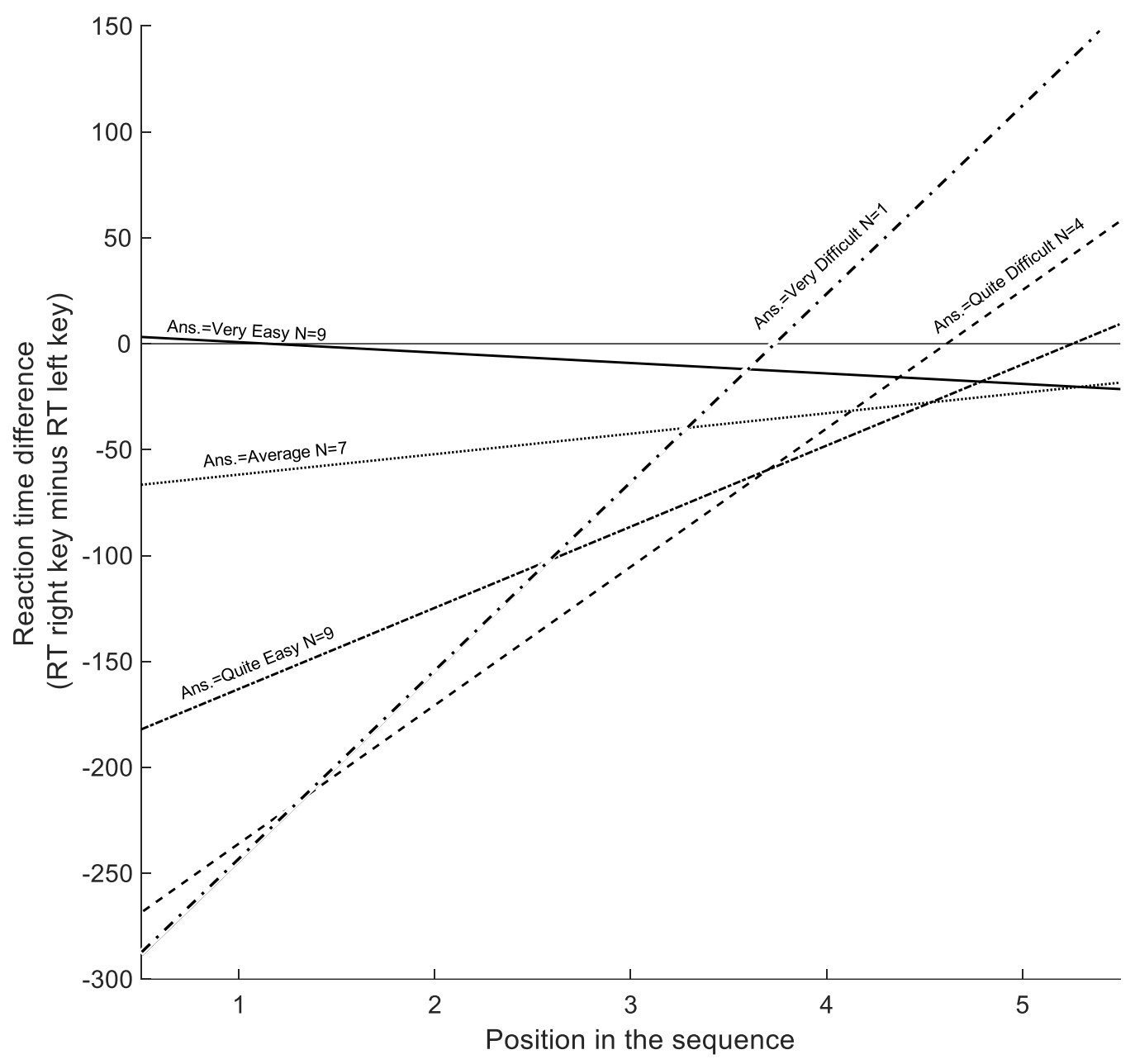

